\title{
INFOBOX 4.2
}

\section{LIGHTNING, COSMIC RAYS AND ENERGETIC PARTICLES}

\author{
Yoav Yair ${ }^{1}$
}

Thunderstorms and lightning are among the fiercest and most beautiful natural phenomena, feared, respected and worshiped by mankind since ancient times. As described in Chapter 4.8, at any given moment, there are a few thousand thunderstorms around the planet, with a global flash rate estimated at 50 strikes per second. Lightning activity depends on the season and geographical location: with the aid of lightning location networks and satellite data, we are now able to map the areas where lightning frequency is greatest. As Figure 1 shows, most lightning occur over the tropical continental regions in Africa, South-East Asia and South-America. Over the oceans, lightning is less frequent, and at high latitudes, it is almost entirely absent. The Inter-tropical Convergence Zone (ITCZ) migrates with the season from the Southern hemisphere summer to the Northern one, a fact that is clearly reflected in the location of lightning activity: almost $90 \%$ of global lightning occurs in the respective summer hemisphere.

The impressive lightning flash rate is a proof of the efficiency by which clouds are being electrically charged and discharged. But, even with our present-day capabilities of advanced computer simulations, sophisticated laboratory experiments and airborne measurements, we are still unsure about the nature of charging processes taking part within the tumultuous heart of a thundercloud. There, trillions of ions, water drops, ice crystals and hail particles collide with each other in subzero temperatures. On average, these collisions charge the particles such that smaller ones carry a net positive charge, and the larger ones negative charge. The difference in fall-speeds in air of particles of different phases, masses and shapes leads to the formation of charged regions with different polarities, making the thundercloud behave like a giant battery (with a "plus" and a "minus", separated by kilometers). When the resultant electric field that is present between the charge centers exceeds a certain threshold value, an electron avalanche starts at some point, and rapidly progresses to form a conductive channel that moves in the air by discrete steps (hence it is called "a stepped leader"). If the process occurs between two adjacent (but opposite) centers within the cloud, we would get

\footnotetext{
${ }^{1}$ School of Sustainability, Interdisciplinary Center Herzliya, Israel
} 


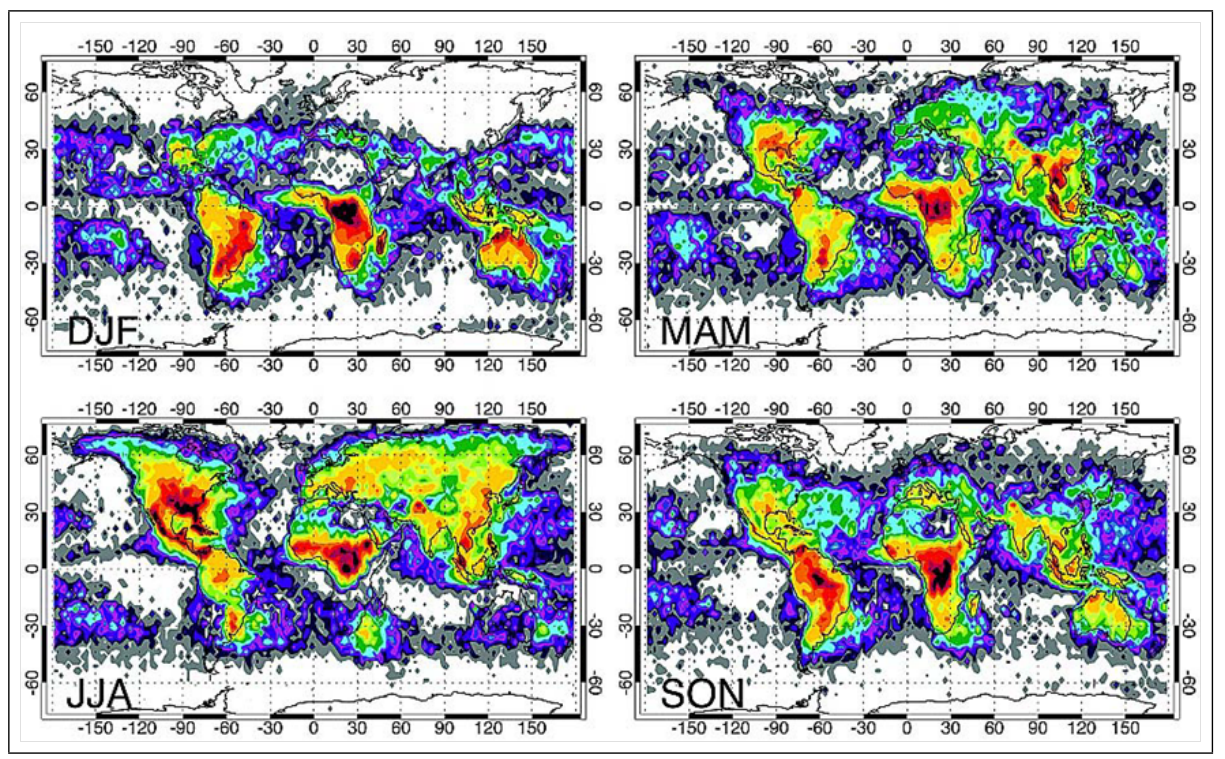

Fig. 1. The seasonal pattern of global lightning activity as observed by the Optical Transient Detector satellite. The different parts represent the three-monthly accumulated data, reflecting the seasonal pattern. Upper left, December-January-February (DJF); Upper right: March-April-May (MAM); Bottom left June-July-August (JJA); Bottom right, September-October-November (SON) ((Christian et al., 2003)). Copyright AGU, reprinted with permission).

an Intra-Cloud flash (IC). If the process moves downward, it forms the familiar chaotic zig-zag pattern until the leader tip attaches to an object on the ground, creating a cloud-to-ground flash (CG). The approaching leader short-circuits the charge center aloft to the surface, and huge amounts of electrons flow downwards, heating the surrounding air to over $30000^{\circ} \mathrm{C}$, making it glow brightly. This is the lightning that we see, and it normally lasts less than half a second. The heated air around the lightning channel rapidly expands, a fact we all recognize as the sound of thunder. The contact point, where the leader attaches, can be a building, a tree or a person, a fact that makes lightning extremely dangerous and sometimes lethal.

There are still many mysteries surrounding the initial breakdown process that ignites a lightning flash. Balloon measurements performed inside developing thunderstorms show that the conventional breakdown field of about $1 \mathrm{MV} \mathrm{m}^{-1}$ is never reached, and that lightning occurs at fields weaker by an order of magnitude. This hints at another process taking place. One possible mechanism is the "Runaway Breakdown" process, in which acceleration of relativistic electrons originating from extensive air-showers (EAS) created by energetic cosmic rays trigger the avalanche, leading to a lightning discharge (Gurevich and Karashtin, 2013). Alternatively, the avalanche may be initiated by proton fluxes during solar proton events (SPE), 
especially those that are energetic enough to reach the lower troposphere (where clouds develop). These effects would likely show a latitudinal dependence, due to the shielding effect of the Earth's magnetic field that determines how many particles would actually penetrate down.

If fluxes of external energetic particles are indeed effective in the initiation of a leader that starts the discharge, then we might search for a signal of enhanced (or reduced) lightning activity in close conjunction with an increase (or decrease) in the flux of cosmic rays / solar energetic particles reaching the atmosphere. If, however, the influence of enhanced energetic particles (primary or secondary) is through slower changes in the ionisation and other charging processes affecting charge separation within thunderclouds, then the effect may be diluted by other microphysical processes or be delayed by hours to days.

Research conducted in the 1980 s attempted to relate cosmic ray maxima (determined from minimum Kp index) with the number of lightning days, obtained from reports of meteorological stations (a "Lightning Day" is defined as a day when an observer sees a lightning flash or hears a thunder). Results suggest that there is a maximum in lightning activity 3 days after the maximum cosmic ray flux (Lethbridge, 1981). Although the exact mechanism is unclear, this correlation does not imply causality and cannot be explained by the direct seeding-ignition hypothesis. It may reflect changes in the ionisation rates, and by inference of the charging efficiency at cloud altitudes.

An opposite effect is expected following a Forbush Decrease (FD, and see Chapter 2.3), identified through neutron counts by the World-Wide Neutron Monitor Network. If there is a link, one can expect a decrease in the amount of lightning following such an event, because a large dip in the number of arriving cosmic ray particles would mean fewer seed electrons available for breakdown. Recent analysis of lightning data obtained from the World-Wide Lightning Location Network (wwlln.net) shows that in strong FD events, a minimum in lightning counts occurred a day after the onset of that event ${ }^{1}$. It should be noted that the WWLLN only detects the strongest cloud-to-ground flashes and has an overall global detection efficiency of about $25 \%$, so the result may apply only to the strongest flashes. The delay is also hard to explain, because it means that the effect is not instantaneous as one would expect if the Runaway Breakdown hypothesis was true.

A study preformed for the continental US derived lightning from the National Lightning Detection Network (Chronis, 2009) and showed a minimum in lightning activity $4-5$ days after a Forbush event. For longer time periods, e.g., monthly statistics of lightning occurrence, that study showed a positive trend between lightning activity and cosmic ray fluxes in Northern Hemisphere winter months (December-January-February), but a non-significant trend in summer. This may

${ }^{1}$ Okike, $\mathrm{O}$ and Collier AB, Testing the cosmic-ray lightning connection hypothesis. 30th URSI General Assembly and Scientific Symposium, Istanbul, Turkey, 13-20 August, 2011, 10.1109/URSIGAS.2011.6051175, IEEE, 2011. 
be due to differences in the relative importance of various charging processes in winter and summer and to the nature of the clouds themselves. Another study, conducted in Europe (Schlegel et al., 2001) related lightning data from local networks in Germany and Austria to solar parameters (sunspot number R, geomagnetic activity Ap and radio flux index F10.7) and to cosmic ray fluxes. In general, they found only weak influence of solar activity on lightning frequency compared to the decisive meteorological factors, and found that the overall effect was opposite to the former study showing high frequency of lightning during times of low cosmic ray flux, i.e., when solar activity was high. The explanation for that result was the operation of planetary-wave dynamics. Still, it is unclear how exactly solar signals are transmitted to the lower atmosphere. In Brazil, a statistical analysis of thunder-day data from 1951 to 2009 in 7 major cities searched for a signal of the 11-year solar cycle in lightning activity (Pinto Neto et al., 2013). The results showed an anti-correlation between solar activity and lightning only in 3 cities (with a weaker one in the other 3 , and none in the last), explained by a "shielding effect" of cosmic rays by the heliosphere in years with large sunspot numbers. A study conducted in south-east Asia failed to find any significant correlation between sunspot numbers, Ap index, the F10.7 index, and lightning (Siingh et al., 2013).

These different and sometimes contradictory results clearly show that there is a geographic variability in the observed correlations between solar variability (affecting cosmic ray fluxes) and lightning, and that it is different on different time scales. It also leaves open the questions on the exact nature of the coupling mechanism(s): is it via the ionization and charging process within the evolving cloud, or via the runaway-breakdown-leader process when the thunderstorms are mature. It may also be that both processes take place, or others still unknown. With the advance in global coverage of lightning flashes, better studies can be made, where new and improved results will help shed light on this intriguing topic.

In recent years, observations from satellites, ground-based sensors and laboratory experiments found intriguing new results showing that lightning discharges are related to energetic particles in a completely different manner. It appears that lightning emits and produces very energetic particles by themselves: X-rays, neutrons, positrons and gamma-rays are all generated by complex processes taking place in the tips of lightning leaders during various stages of the breakdown process. These new results imply that we are still far from a complete understanding of this wonderful natural phenomenon, and that there is a lot to be discovered. 